\title{
Estudio sobre la desalineación de montaje óptima del árbol superior de los molinos de caña de azúcar
}

\author{
Nelson Arzola*\$, Rafael Goytisolo** \\ * Departamento de Ingeniería Mecánica y Mecatrónica, Facultad de Ingeniería, \\ Universidad Nacional de Colombia \\ ** Departamento de Ingeniería Mecánica, Facultad de Ingeniería Mecánica, \\ Universidad de Cienfuegos, Cuba \\ §e-mail:narzola@unal.edu.co
}

\section{Resumen}

Se realiza un estudio sobre la influencia de la desalineación de montaje en la vida de fatiga del árbol superior de los molinos de caña de azúcar. Se determina la razón de propagación de las grietas de fatiga en la sección más propensa a este tipo de falla, a partir del análisis de la variación de las cargas considerando la flotación del árbol superior durante la operación del molino. Es posible aumentar la vida de fatiga de los árboles empleando el procedimiento descrito, el cual determina un valor óptimo de desalineación de montaje. Para el molino estudiado se obtiene una desalineación de montaje óptima de valor igual a $1 \mathrm{~mm}$ por debajo del árbol de salida del reductor de baja. Para esta posición, el árbol superior alcanza una vida de fatiga de 1189 días de operación. Un análisis de vida de fatiga similar puede ser realizado para los árboles inferiores del molino.

Palabras clave: Desalineación, Mecánica de fractura, Molino, Fatiga.

\section{Study on the optimal assembly misalignment of sugar-mill top shafts}

\begin{abstract}
A study on the influence of misalignment in the fatigue life of sugar-mill top shafts is presented. The analysis of the behavior of the shaft loads, considering the flotation during the operation of the mill, allows determining the crack growth rate in the most critical section. It is possible to increase the fatigue life of the top shaft using this approach, which determines a good value of assembly misalignment. For the mill studied here, an optimal assembly misalignment of $1 \mathrm{~mm}$, between the top shaft and the gear box reducer shaft, was obtained. For this position, the shaft fatigue life is 1,189 days of operation. A similar analysis of fatigue life can be carried out for the bottom shafts of the mill.
\end{abstract}

Keywords: Misalignment, Fracture mechanic, Mill, Fatigue. 


\section{Introducción}

En la mayoría de los molinos de caña de azúcar actuales la transmisión de potencia se realiza utilizando acoplamientos cuadrados. La ventaja de estos acoplamientos, frente a otros tipos, radica en su relativo bajo costo y fácil diseño; pero poseen como inconveniente, una limitada capacidad para absorber la desalineación que se produce entre el árbol de salida del reductor de baja del tren de engranes y el árbol superior del molino. Varios autores han descrito la influencia perjudicial que provocan las cargas complementarias, que surgen en la espiga cuadrada producto de la desalineación, en la vida de fatiga de los árboles de molinos (Okamura et al.,1972; Muñoz \& Lewinski, 1996; Tosio, 1992).

Sin embargo, no se ha realizado ningún estudio que modele o describa analíticamente este fenómeno. En esta investigación se emplean elementos novedosos, como son un nuevo esquema de análisis que considera el árbol superior como una viga sobre fundación elástica, y un modelo matemático que relaciona las cargas de contacto en la espiga cuadrada en función de la potencia transmitida y la flotación en operación del árbol superior, para el cálculo del estado tensional presente en la media caña del collarín más cercano al acoplamiento. Esta sección es la más crítica en cuanto a falla por fatiga, teniendo el mayor porcentaje de roturas. Otro elemento nuevo es la aplicación del modelo fractomecánico de propagación de la grieta.
El modelo de grieta seleccionado para los cálculos es el de grieta circunferencial, que aunque no es la más probable que surja, si es la más crítica por su mayor velocidad de propagación y su menor tamaño crítico para provocar la falla por rotura frágil de los árboles de molinos.

El sector industrial azucarero posee evidencia empírica sobre la relación existente entre la disminución de la vida de fatiga del árbol superior y la magnitud de la desalineación de montaje del árbol superior. En este artículo, se aborda esta problemática con el fin de demostrar de manera teórica como se correlacionan estos dos fenómenos, brindando además un procedimiento de cálculo del valor de desalineación óptimo para maximizar la vida de fatiga del árbol superior.

En la actualidad, la vida de fatiga de un árbol superior de molino se encuentra usualmente entre 400 y 700 días de operación, lo cual trae asociado un costo considerable para la industria azucarera por concepto de reparación. El costo de un árbol de molino es de alrededor de US \$ 10000 , en dependencia de sus características geométricas y proveedor. Al costo total de la rotura se adiciona el costo indirecto por parada forzosa del proceso de molienda, el cual es varias veces superior.

\section{Metodología}

En la Figura 1 se muestra la solución empleada para transmitir la potencia hacia el árbol superior del molino de caña de azúcar.

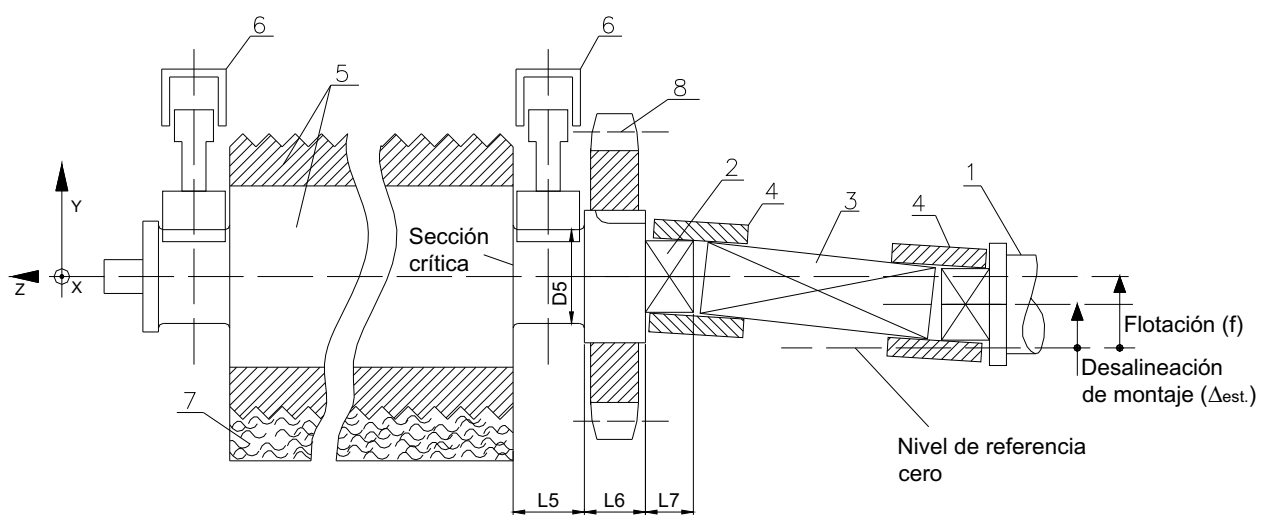

Figura 1. Diagrama del árbol superior donde se muestra su posición con relación a la espiga cuadrada de salida del reductor de baja. 
La espiga cuadrada de salida del reductor de baja (1) transmite la potencia hacia la espiga cuadrada del árbol superior (2) por medio del árbol cuadrado (3) y los acoplamientos cuadrados (4). El conjunto árbol maza superior (5) es empujado hacia abajo por medio de cilindros hidroneumáticos (6) colocados sobre los collarines del árbol superior, provocando la compresión del colchón de bagazo (7) que es forzado a pasar, por medio del arrastre generado por la rotación de las mazas, a través del espacio formado entre la maza superior y las dos mazas inferiores (que no se muestran en la figura). De esta manera se logra la extracción del jugo de la caña de azúcar. El engrane o corona superior (8) transmite el movimiento de rotación a los engranes o coronas inferiores, acoplados a los árboles inferiores.
Cuando el molino no posee alimentación, la maza superior se encuentra en el nivel de referencia cero, pero una vez que comienza a pasar el colchón de bagazo, la maza superior se levanta hasta la posición de trabajo normal. Si el reglaje (setting) del molino se realiza para una desalineación de montaje igual a la flotación media del árbol superior, entonces coinciden los ejes de simetría de este último y del árbol de salida del reductor de baja.

Empleando el esquema de viga sobre fundación elástica (Arzola, 2003), es posible determinar las fuerzas internas que actúan en la sección de cambio en diámetro del collarín más próximo a la corona, para el árbol superior. Las fuerzas de cortante $\left(V_{\mathrm{x}}\right.$ y $\left.V_{\mathrm{y}}\right)$ y momentos flectores $\left(M_{\mathrm{x}}\right.$ y $\left.M_{\mathrm{y}}\right)$ están dadas por las expresiones:

$V_{y}=\left(q_{w 5}^{y}+q_{w c 2}^{y}+q_{h i d 2}\right) L_{5}+\left(q_{w g s}^{y}+q_{w 6}^{y}-q_{n c c}^{y}+q_{n c b}^{y}\right) L_{6}+q_{w 7}^{y} L_{7}-P_{a}+P_{b}$

$$
\begin{aligned}
M_{x}=- & \left(q_{w 5}^{y}+q_{w c 2}^{y}+q_{\text {hid } 2}\right) \frac{L_{5}^{2}}{2}-\left(q_{w g s}^{y}+q_{w 6}^{y}+q_{n c b}^{y}-q_{n c c}^{y}\right) L_{6}\left(L_{5}+\frac{L_{6}}{2}\right)-q_{w 7}^{y} L_{7}\left(L_{5}+L_{6}+\frac{L_{7}}{2}\right) \\
& +P_{b}\left(L_{5}+L_{6}+\frac{L_{7}-I_{1}}{2}\right)-P_{a}\left(L_{5}+L_{6}+\frac{L_{7}+I_{1}}{2}\right)-\mu_{c}\left(P_{a}+P_{b}\right) \frac{I_{3}}{2}
\end{aligned}
$$

$V_{x}=\left(q_{w 5}^{x}+q_{w c}^{x}-q_{R d e r}\right) L_{5}+\left(q_{n c c}^{x}+q_{n c b}^{x}+q_{w g s}^{x}+q_{w 6}^{x}\right) L_{6}+q_{w 7}^{x} L_{7}$

$$
\begin{aligned}
M_{y}= & \left(q_{R d e r}-q_{w c}^{x}-q_{w 5}^{x}\right) \frac{L_{5}^{2}}{2}-\left(q_{n c c}^{x}+q_{n c b}^{x}+q_{w g s}^{x}+q_{w 6}^{x}\right) L_{6}\left(L_{5}+\frac{L_{6}}{2}\right)-q_{w 7}^{x} L_{7}\left(L_{5}+L_{6}+\frac{L_{7}}{2}\right) \\
& +\left(P_{a}+P_{b}\right) \frac{\mu_{c} I_{3}}{2}
\end{aligned}
$$


donde $q_{w i}^{y}(\mathrm{kN} / \mathrm{m})$ es el peso específico por unidad de longitud del escalón $i$ del árbol en la dirección del eje $y ; q_{w c 2}^{y}(\mathrm{kN} / \mathrm{m})$ es la componente (en la dirección del eje $y$ ) de la carga sumaria del peso de la chumacera, fricción de la chumacera con la virgen y fricción de la zapatilla con la pared interior del cilindro para el lado derecho; $q_{n c c}^{y}, q_{n c c}^{x}$ $(\mathrm{kN} / \mathrm{m})$ son las fuerzas distribuídas que actúan sobre el árbol superior debido a la transmisión del torque por la corona cañera; $q_{n c b}^{y}, q_{n c b}^{x}(\mathrm{kN} / \mathrm{m})$ son las fuerzas distribuídas que actúan sobre el árbol superior debido a la transmisión del torque por la corona bagacera; $q_{\text {wgs }}^{y}(\mathrm{kN} / \mathrm{m})$ es la componente (en la dirección del eje $y$ ) del peso propio de la corona superior; $q_{\text {Rder }}(\mathrm{kN} / \mathrm{m})$ es la reacción de la virgen sobre la chumacera; $P_{a}, P_{b}(\mathrm{kN})$ son las fuerzas de contacto en la espiga cuadrada del árbol superior ; $L_{5}, L_{6}, L_{7}(\mathrm{~m})$ son las longitudes de la chumacera, el asiento para la corona y la espiga cuadrada respectivamente; $\mu_{\mathrm{c}}$ es el coeficiente de fricción entre la espiga y el acoplamiento cuadrado; $l_{3}(\mathrm{~m})$ es el lado de la espiga cuadrada; $l_{1}(\mathrm{~m})$ es la distancia entre los puntos de contacto en el acoplamiento cuadrado.

La distancia entre los puntos de contacto en el acoplamiento cuadrado se determina por (Okamura, et al., 1972):

$$
I_{1}=6.5|\lambda| L_{c}
$$

donde $\lambda$ es la razón de excentricidad y $L_{c}$ (m) es la longitud del acoplamiento cuadrado.

El momento torsor en la sección bajo estudio se calcula por la expresión siguiente: donde $\mu_{\text {st_b }}$ es el coeficiente de fricción acerobronce, $\varphi_{M C}$ es la fracción de la potencia total que se transmite a la maza cañera, $\varphi_{M B}$ es la fracción de la potencia total que se transmite a la maza bagacera y $D_{5}(\mathrm{~m})$ es el diámetro de la chumacera.

El coeficiente de fricción acero-bronce en las chumaceras del árbol superior se determina utilizando los resultados experimentales que relacionan este parámetro con la velocidad de deslizamiento y la presión específica sobre la chumacera (Lafargue \& Rodríguez, 2001).

Con base en los resultados experimentales de Okamura et al. (1972), se obtuvo un modelo matemático que relaciona las fuerzas que se generan en la espiga cuadrada del árbol superior con la potencia transmitida y la flotación, las cuales se expresan como sigue:

$$
\begin{aligned}
& P_{a}=\frac{30 N_{m}}{\pi n}\left(\frac{1}{I_{3}} \pm \frac{A_{0}}{2}\right) \\
& P_{b}=\frac{30 N_{m}}{\pi n}\left(\frac{1}{I_{3}} \mp \frac{A_{0}}{2}\right)
\end{aligned}
$$

donde

$$
A_{0}=1.143+955.063|\lambda|-33441.9 \lambda^{2}
$$

$N_{m}(\mathrm{~kW})$ es la potencia transmitida por la espiga cuadrada del árbol superior, $n$ (rpm) es la velocidad de rotación de los árboles del molino y $A_{o}\left(m^{-1}\right)$ es el factor que tiene en cuenta la razón de excentricidad.

La razón de excentricidad puede calcularse por medio de la Ec. (10):

$$
T=\frac{\left(P_{a}+P_{b}\right) I_{3}}{2}\left[1-\left(\varphi_{M C}+\varphi_{M B}\right)\right]-\mu_{s t_{-} b} \frac{D_{5} L_{5}}{2} \sqrt{\left(q_{R d e r}+q_{w c 2}^{x}\right)^{2}+\left(q_{\text {hid } 2}+q_{w c 2}^{y}\right)^{2}}
$$




$$
\lambda=\frac{f-\Delta_{\text {est }}}{L_{b}}
$$

donde $f(\mathrm{~mm})$ es la flotación media del árbol superior, $\Delta_{\text {est }}(\mathrm{mm})$ es la desalineación de montaje y $L_{b}(\mathrm{~mm})$ es la longitud de la barra cuadrada.

Las fuerzas complementarias son reducidas a un mínimo cuando el árbol de salida del reductor de baja está instalado de tal forma que se alcanza la mínima desalineación con el árbol superior durante la operación del molino. Pero esta no es la condición para que se alcance el estado tensional más favorable en el cambio en diámetro del collarín más próximo a la corona, debido a que existen otras cargas actuando entre dicha sección y la espiga cuadrada.

El estado tensional alcanzado para un punto cualquiera de la sección bajo análisis del árbol es triaxial, existiendo una tensión normal producto de los momentos flectores, calculados por las Ecs. (2) y (4), y unas tensiones tangenciales de direcciones radial y circunferencial, debidas a las fuerzas de cortante $y$ al momento torsor, calculadas por las Ecs. (1), (3) y (6), respectivamente.

Las tensiones en un punto cualquiera de la sección se determinan mediante las siguientes expresiones:

$\sigma_{N(\rho, \varphi)}=10^{-3}\left[\frac{64}{\pi D_{5}^{4}} \rho\left(M_{x} \sin \varphi+M_{y} \cos \varphi\right) \pm \frac{4 N}{\pi D_{5}^{2}}\right]$

$\tau_{r(\rho, \varphi)}=10^{-3}\left[\frac{64\left(\frac{D^{2}}{4}-\rho^{2}\right)}{3 \pi D_{5}^{4}}\left(V_{x} \cos \varphi+V_{y} \sin \varphi\right)\right]$

$\tau_{t(\rho, \varphi)}=10^{-3}\left[\frac{16}{3 \pi D_{5}^{2}}\left(V_{y} \cos \varphi-V_{x} \sin \varphi\right)+\frac{32 T}{\pi D_{5}^{4}} \rho\right]$

donde $\sigma_{N(\rho, \theta)}(\mathrm{MPa})$ es la tensión normal, $\tau_{r(\rho, \theta)}$ $(\mathrm{MPa})$ es la tensión tangencial radial, $\tau_{t(\rho, \theta)}(\mathrm{MPa})$ es la tensión tangencial circunferencial, $M_{\mathrm{x}}, M_{\mathrm{y}}$ $(\mathrm{kN} \mathrm{m})$ son los momentos flectores actuando en los ejes $x$ y $y$, respectivamente, $N(\mathrm{kN})$ es la fuerza de tracción o compresión que actúa sobre la sección transversal, $V_{\mathrm{x}}, V_{\mathrm{y}}(\mathrm{kN} \mathrm{m})$ son las fuerzas de cortante que actúan en los ejes $x$ y $y$, respectivamente, $\rho(\mathrm{m}), \varphi\left({ }^{\circ}\right)$ son las coordenadas polares de posición del punto bajo análisis.

El proceso de fatiga que se desarrolla en la zona bajo estudio es de fatiga multiaxial bajo cargas no proporcionales, el cual es difícil de modelar de forma precisa utilizando los procedimientos clásicos de cálculo. Por tal motivo, se selecciona un análisis de crecimiento subcrítico de grietas aplicando conceptos de mecánica de la fractura. La ecuación de crecimiento de la grieta escogida es la de Paris (Dowling, N., 1999), que emplea un factor de intensidad de tensiones equivalente que tiene en cuenta los dos modos de carga presentes (modos I y III) para una grieta circunferencial:

$$
\frac{d a}{d N}=C\left(\Delta K_{e q}\right)^{n}
$$

donde

$\Delta K_{\text {eq }}=\left[\left\langle\sqrt{K_{I_{(\varphi)}^{2}+\frac{1}{1-\mu} K_{I I(\varphi)}^{2}}}\right\rangle_{\text {max }}\left\langle\sqrt{K_{I_{(\varphi)}^{2}+\frac{1}{1-\mu} K_{I I(\varphi)}^{2}}^{2}}\right\rangle_{\text {min }}\right] \sqrt{\pi a}$

$\frac{d a}{d N} \quad(\mathrm{~m} /$ ciclo) es el incremento del tamaño de la grieta para un ciclo, $\Delta K_{e q}\left(\mathrm{MPa} \mathrm{m}^{1 / 2}\right)$ es la variación del factor de intensidad de tensiones equivalente, $C, n$ son coeficientes que dependen del material y de la razón de asimetría del ciclo, $K_{I(\varphi)}, K_{I I(\varphi)}$ $\left(\mathrm{MPa} \mathrm{m}^{1 / 2}\right)$ son los factores de intensidad de tensiones para los modos I y III de carga, respectivamente, $a(\mathrm{~m})$ es la profundidad de la grieta y $\mu$ es el coeficiente de Poisson.

El tiempo de propagación subcrítico de la grieta desde un tamaño inicial de $50 \mathrm{~m}$ hasta el tamaño crítico es calculado por medio de la Ec. (14). La determinación de estos tamaños se realiza considerando las propiedades fractomecánicas del material, modelo de grieta y estado tensional presente en la sección de análisis (Arzola, 2003). 


\section{Resultados y discusión}

La razón de excentricidad se calcula por la Ec. (10) para un grupo de valores de desalineación de montaje, de modo que pueda determinarse la influencia que éste posee en la vida de los árboles de los molinos.
En la Figura 2 se muestra el comportamiento de las fuerzas internas en la sección bajo análisis con la flotación del árbol superior. El valor de desalineación de montaje es $12 \mathrm{~mm}$, para el molino analizado. Como se aprecia, existe una variación bastante acentuada de las fuerzas internas con la flotación que posee el árbol superior.

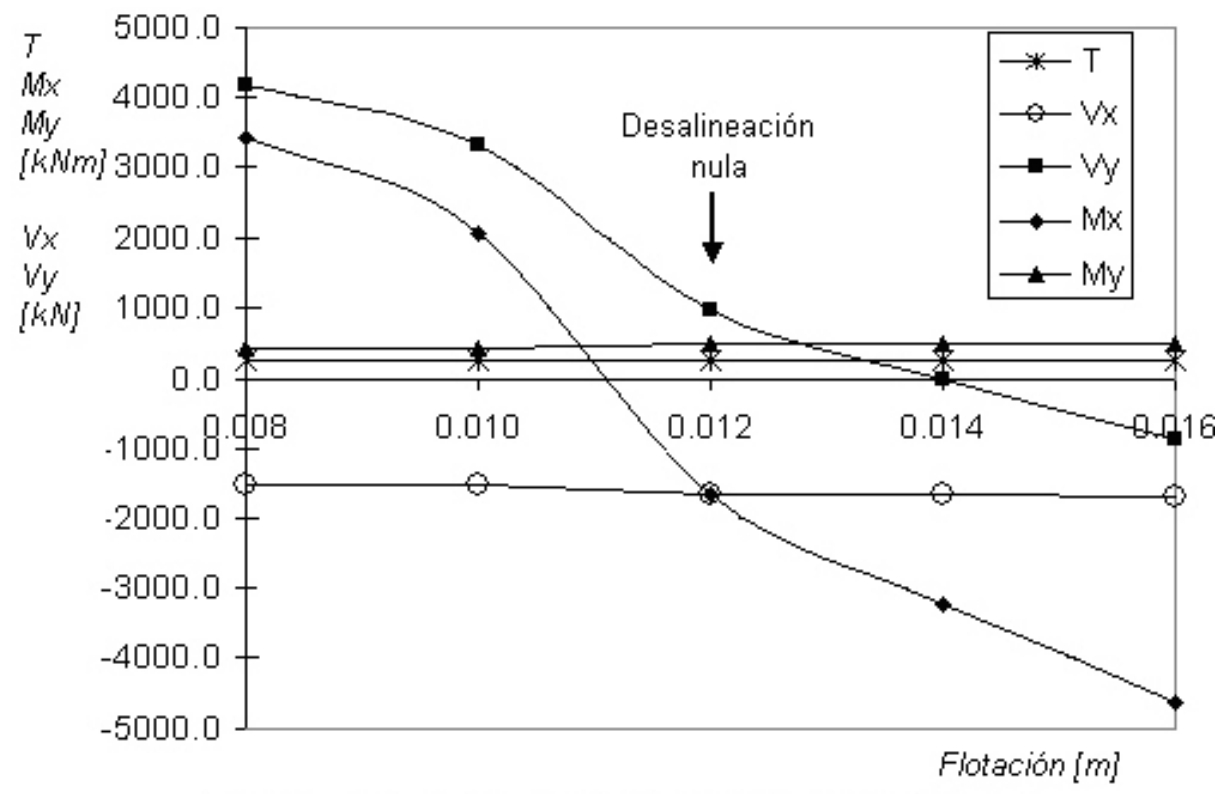

Figura 2. Comportamiento de las fuerzas internas con la flotación para la sección analizada.

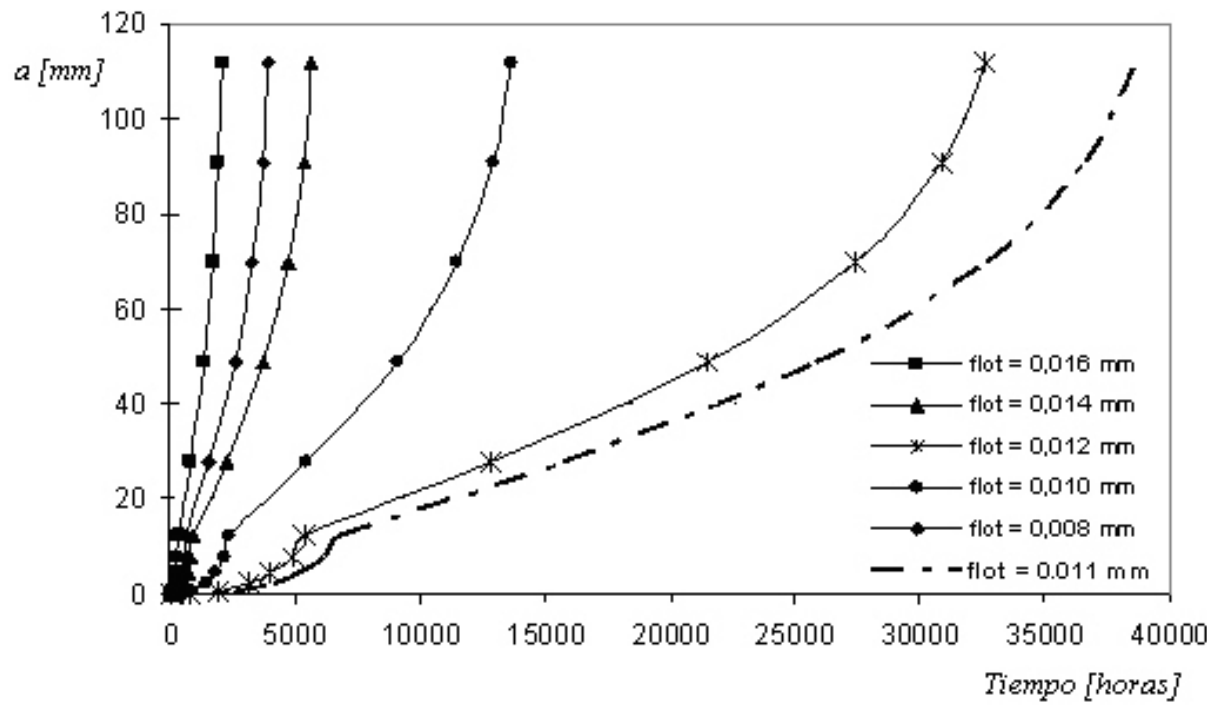

Figura 3. Predicción de la propagación de la grieta circunferencial para distintos valores de flotación. 
Al utilizar el modelo de propagación de grieta circunferencial presentado, en el cálculo de las tasas de crecimiento según las distintas condiciones de flotación del árbol superior, se puede conocer el grado de criticidad que posee cada posición de flotación del árbol superior.

En la Figura 3 se muestra una familia de curvas de propagación de grietas circunferenciales para distintos valores de la desalineación de montaje.

En la Figura 4 se muestran los valores de tiempo de propagación de estas grietas desde un microdefecto inicial hasta el tamaño crítico. La curva representa la línea de tendencia del comportamiento de la vida de fatiga del árbol.

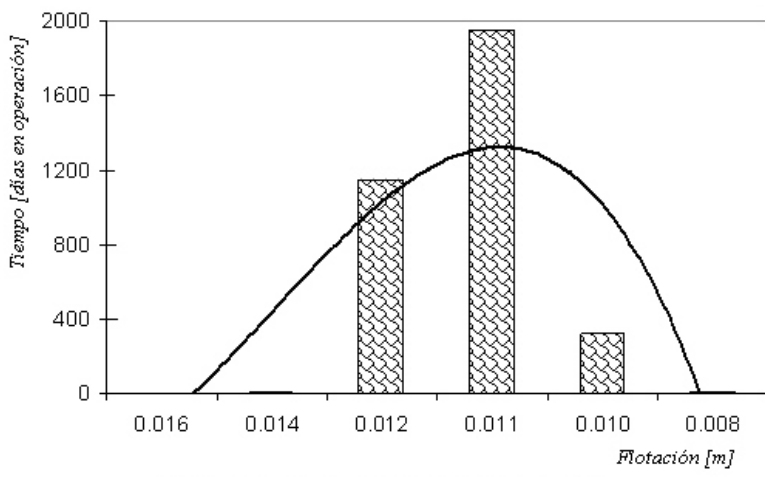

Figura 4. Comportamiento de la vida de fatiga con la flotación del árbol superior del molino.

La durabilidad máxima del árbol superior del molino de caña de azúcar analizado se alcanza para una razón de desalineación de $\lambda=-0.00095$, la cual ocurre para una posición del árbol superior de $1 \mathrm{~mm}$ por debajo del árbol de salida del reductor de baja. Amedida que la desalineación de montaje se aleja de este valor, las fuerzas internas en la sección analizada aumentan, lo cual trae como consecuencia un incremento de la tasa de crecimiento de las grietas circunferenciales. Este valor de desalineación de montaje óptimo se alcanza mediante iteraciones sucesivas realizadas para la vida de fatiga del árbol superior en un entorno cercano a la posición concéntrica del árbol superior y el árbol de salida del reductor de baja, hasta que se alcanza el valor máximo de vida de fatiga del elemento bajo análisis.
Los resultados obtenidos están en concordancia con la vida de fatiga de varios árboles superiores de molino que han fallado bajo distintas condiciones de desalineación de montaje. Lamentablemente, las condiciones de registro y control que prevalecen en la industria azucarera son insuficientes para validar estos resultados de manera totalmente confiable. Por ello, se recomienda un modo de validación de los resultados que emplee un modelo a escala del sistema formado por el árbol superior, acoplamiento cuadrado y salida del reductor de baja, con el cual se controlen y registren de una manera más precisa los valores de desalineación, tensión-deformación y propagación de grieta.

\section{Conclusiones}

- La durabilidad de los árboles de los molinos de caña de azúcar está marcada en gran medida por la desalineación que posea el árbol superior con respecto al árbol de salida del reductor de baja. Una flotación, positiva o negativa, excesiva provoca la rotura por fatiga prematura de estos elementos, de modo que es imprescindible desde el punto de vista técnico - económico un correcto montaje relativo entre el árbol de salida del reductor de baja y el árbol superior.

- El valor óptimo de la desalineación de montaje, desde el punto de vista de la durabilidad de los árboles, deberá calcularse para cada molino en particular teniendo en cuenta los parámetros constructivos del molino, su ajuste (setting) y las cargas que actúan en el mismo.

- Para el molino analizado en el trabajo resultó que la desalineación de montaje óptima se alcanza cuando el árbol superior se encuentra en una posición de $1 \mathrm{~mm}$ por debajo del árbol de salida del reductor de baja. Para esta posición la vida total del árbol es igual a 1189 días de operación.

- Se produce una disminución marcada de la vida de fatiga de los árboles superiores cuando el valor de desalineación, positiva o negativa, aumenta; hasta el caso extremo para el cual con una desalineación de $+4 \mathrm{~mm}$ la vida de fatiga se reduce hasta 14 días de operación. 
- Es posible realizar un análisis de vida de fatiga similar para los árboles inferiores del molino, demostrándose que la vida de los mismos también se ve afectada de forma similar por la magnitud de la desalineación.

\section{Referencias bibliográficas}

Arzola, N. (2003). Esquema de análisis y aplicación de la mecánica de la fractura a la falla por fatiga de los árboles de los molinos de caña de azúcar. Tesis de Doctorado, Universidad de Cienfuegos, Cuba.

Dowling, N. (1999). Mechanical behaviour of materials: engineering methods for deformation, fracture, and fatigue. Upper Saddle River (NJ): Prentice Hall, Inc.
Lafargue, F., \& Rodríguez, C. (2001). Influencia de la velocidad de deslizamiento en el consumo de potencia de las chumaceras de los cilindros de caña, Revista Centro Azúcar, 5 (3), 33-36.

Muñoz, G., \& Lewinski J. (1996). Análisis del comportamiento mecánico del molino cañero. International Sugar Journal 98 (1172), 386-390.

Okamura, H., Tanaka, H., \& Terao, M. (1972). Square box couplings in cane mill drives, Parts I and II. International Sugar Journal 74 (886), 291-293; 74 (887), 323-327.

Tosio, C.T. (1992). Sugar Mill Drive Couplings an alternative designs. Proceedings of the 21st Congress of the International Sugar Cane Technologists. Vol. 3, p. 807-817, Bangkok, Thailand. 